\title{
Experimental Results on Low Alpha Electron-Storage Rings*
}

\author{
D. Robin \\ Advanced Light Source, Accelerator and Fusion Research Division \\ Lawrence Berkeley National Laboratory, University of California, Berkeley, CA 94720
}

H. Hama

UVSOR Facility, Institute for Molecular Science, 38 Myodaiji, Okazaki 444, Japan

A. Nadji

LURE, Centre Universitaire de Paris-Sud, Bat. 209A, 91405 Orsay Cedex, France

September 1995

Paper Presented at the a Workshop on the Production, Measurement, and Applications of Short Bunches of Electrons and Positrons in Linacs and Storage Rings, Brookhaven National Lab,

September 28-30, 1995.

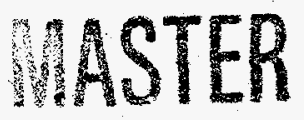

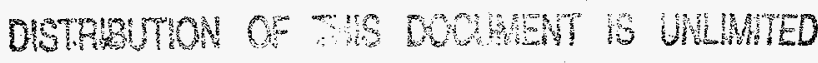

*This work was supported by the Director, Office of Energy Research, Office of Basic Energy Sciences, Materials Sciences Division of the U.S. Department of Energy, under Contract No. DE-AC03-76SF00098 


\section{DISCLAIMER}

This report was prepared as an account of work sponsored by an agency of the United States Government. Neither the United States Government nor any agency thereof, nor any of their employees, make any warranty, express or implied, or assumes any legal liability or responsibility for the accuracy, completeness, or usefulness of any information, apparatus, product, or process disclosed, or represents that its use would not infringe privately owned rights. Reference herein to any specific commercial product, process, or service by trade name, trademark, manufacturer, or otherwise does not necessarily constitute or imply its endorsement, recommendation, or favoring by the United States Government or any agency thereof. The views and opinions of authors expressed herein do not necessarily state or reflect those of the United States Government or any agency thereof. 


\section{DISCLAIMER}

Portions of this document may be illegible in electronic image products. Images are produced from the best available original document. 


\title{
Experimental Results on Low Alpha Electron-Storage Rings ${ }^{1}$
}

\author{
D. Robin \\ Lawrence Berkeley National Labonatory, Berkeley, CA 94720, USA \\ H. Hama \\ UVSOR Facility, Institute for Molecular Science, 38 Myodaiji, Okazaki 444, Japan \\ A. Nadji \\ LURE, Centre Universitaire de Paris-Sud, Bât. 209 A, 91405 Orsay Cedex, France
}

\begin{abstract}
.
We report on experiments performed in two synchrotron light sources, UVSOR and Super-ACO, where the momentum compaction factor is reduced in order to reduce the bunch length. By controlling the second-order momentum compaction factor, UVSOR and Super-ACO have managed to reduce the firstorder momentum compaction factor by 100 . At low current the resulting bunch lengths are less than $10 \mathrm{ps,} \mathrm{a} \mathrm{factor} \mathrm{of} 10$ smaller than normal. Measurements of current dependent bunch lengthening in UVSOR are presented and the cause of the bunch lengthening is determined to be potential-well distortion. We also show that by operating with a negative momentum compaction factor, Super$A C O$ has achieved shorter bunch lengthening and higher peak currents than at positive momentum compaction.
\end{abstract}

\section{INTRODUCTION}

An electron storage ring which produces submillimeter long bunches is a powerful scientific tool, as a synchrotron light source, a damping ring and as a collider. As a synchrotron light source the short bunches, while passing through bending magnets, will produce short light pulses that will improve the time resolution for time of flight experiments. In addition short bunches can generate high power coherent radiation (1-3). As a damping ring the bunch length can be matched to the phase acceptance of the linac, thus avoiding the need for longitudinal bunch compression. As an electron-positron collider shorter bunch lengths. would enable one to decrease the beta function at the interaction point and thus increase the luminosity.

One way to produce short bunches in a storage ring is by reducing the first-order momentum compaction factor, $\alpha_{1}$. The bunch length is proportional to the square root of $\alpha_{1}$. Beyond decreasing the bunch length, reducing $\alpha_{1}$ (and even making

\footnotetext{
${ }^{1}$ This work was supported by the Director, Office of Energy. Research, Office of Basic Energy Sciences, Materials Sciences, Division of the U.S. Department of Energy, under Contract No. DEAC03-76SF00098.
} 
it negative ) promises to enhance the peak current that can stably circulate in a storage ring $(4,5)$. Also very low (nearly isochronous) $\alpha_{1}$ rings if used as drivers for free electron lasers (FELs) promise to provide enhanced longitudinal brilliance, and a large FEL gain (6).

During the last decade several laboratories have attempted to reduce $\alpha_{1}$ in synchrotron light sources (7) and the results have been very encouraging. In this paper we report on some of these results particularly those at UVSOR, Super-ACO and ALS. But first let us start with a discussion about the momentum compaction factor, $\alpha$ and how it effects the bunch length.

\section{MOMENTUM COMPACTION FACTOR}

The difference in path length, $\Delta L$, that an arbitrary particle and the "ideal" particle take to orbit the ring is related by the momentum compaction factor, $\alpha$, to the difference in energy, $\Delta E$, between the arbitrary particle and the "ideal" particle, and is defined as

$$
\frac{\Delta L}{L_{o}}=\alpha \frac{\Delta E}{E_{o}}
$$

where $L_{0}$ is the orbit length and $E_{o}$ is the energy of the ideal particle. The momentum compaction factor is itself a function of $\Delta E / E_{o}$ and can be expressed as a power series expansion in $\Delta E / E_{\mathrm{o}}$ :

$$
\alpha=\alpha_{0} \frac{1}{\left(\Delta E / E_{0}\right)}+\alpha_{1}+\alpha_{2}\left(\Delta E / E_{0}\right)+\alpha_{3}\left(\Delta E / E_{0}\right)^{2}+\ldots
$$

where $\alpha_{0}, \alpha_{1}, \alpha_{2}$, and $\alpha_{3}$ are the zeroeth, first, second and third-order momentum compaction factors respectively.

The bunch length in a storage ring, $\sigma_{b}$, is proportional to the first-order momentum compaction factor, $\alpha_{1}$, and the beam energy spread, $\sigma_{e}$, and is inversely proportional to the the synchrotron tune, $\nu_{s}(8)$ :

$$
\sigma_{b}=\frac{\alpha_{1} R_{0}}{\nu_{s}} \frac{\sigma_{e}}{E_{o}}
$$

where $R_{0}$ is the average ring radius. The synchrotron tune, $\nu_{s}$, is in turn proportional to the square root of $\alpha_{1}$,

$$
\nu_{s}=\frac{1}{2 \pi} \sqrt{\frac{\alpha_{1} e T_{0}}{E_{0}} \frac{d V}{d t}}
$$

where $V_{R F}$ is the RF cavity voltage and $T_{o}$ is the revolution time. So $\sigma_{b}$ is proportional to the square root of $\alpha_{1}$,

$$
\sigma_{b} \propto \sqrt{\left|\alpha_{1}\right|}
$$

and thus can be shortened by reducing the $\alpha_{1}$.

\section{First-Order Momentum Compaction Factor}

The first-order momentum compaction, $\alpha_{1}$, is a function of the ring's local radius of curvature, $\rho$, and the dispersion, $\eta$, and can be expressed as 


$$
\alpha_{1}=\int_{0}^{L_{o}} \frac{\eta}{\rho} \mathrm{ds}
$$

Because $\rho$ is infinite everywhere except in the bending dipoles, only the dipoles will contribute to the integral. Typically in storage rings, the dispersion in the bends and the bending radii are positive so $\alpha_{1}$ is usually positive. The size of $\alpha_{1}$ tends to become smaller as the amount of horizontal focusing increases. As an example, in the smooth approximation (8), $\alpha_{1}$ scales inversely proportional to the square of the horizontal betatron tune, $\nu_{x}$ :

$$
\alpha_{1} \approx \frac{1}{\nu_{x}^{2}}
$$

Equation 7 suggests that large rings with very strong horizontal focussing tend to have naturally small values of $\alpha_{1}$.

Examination of equation 6 reveals that there are two parameters which can be varied in order to reduce $\alpha_{1}$ - even making $\alpha_{1}$ negative. The first parameter is the dispersion, $\eta$, which can be adjusted to be negative in the bends. The second parameter is the bending radius, $\rho$, that can also be made negative by introducing inverted bends. Most existing storage rings do not have inverted bends. In these rings the only way to reduce $\alpha_{1}$ is to make the dispersion negative. This can be accomplished by changing quadrupole field strengths.

To first-order in the change of a quadrupole's integrated field strength, $\Delta k_{l}$, the change in $\alpha_{1}$ is given as

$$
\Delta \alpha_{1}=-\frac{\Delta k_{l}}{L_{o}}\left(\eta_{k x}^{2}-\eta_{k y}^{2}\right)
$$

where $\eta_{k x}$ and $\eta_{k y}$ are the horizontal and vertical values of the dispersion respectively at the location of the quadrupole. So quadrupoles at large values of $\eta_{k x}$ and $\eta_{k y}$ are very effective at changing $\alpha_{1}$.

\section{How Small Can $\alpha_{1}$ Be?}

As $\alpha_{1}$ is decreased, care must be taken to insure that the beam does not become unstable. It is important to maintain a sufficiently large energy acceptance as well as reducing magnet power supply jitter. We now discuss stability criterions.

$$
\text { Size of the Energy Acceptance, } \delta_{m}
$$

The energy acceptance of the machine, $\delta_{m}$, needs to be large enough to produce a long Touschek (9) and quantum (8) beam lifetime. The size of the other momentum compaction factor terms, $\alpha_{0}, \alpha_{2}, \alpha_{3}, \ldots$, effect how small $\alpha_{1}$ can be made while still maintaining a large $\delta_{m}$. In the case of large $\alpha_{1}, \delta_{m}$ is defined as

$$
\delta_{m}=\sqrt{\frac{2 e V_{0}}{\pi h \alpha_{1} E_{o}}\left[\left(\frac{\pi}{2}-\phi_{0}\right) \sin \left(\phi_{o}\right)-\cos \left(\phi_{0}\right)\right]}
$$

where $e$ is the electron charge, $V_{0}$ is the peak RF cavity voltage, $h$ is the harmonic number and $\phi_{0}$ is synchronous phase of the electrons.

As $\alpha_{1}$ is reduced, $\delta_{m}$ defined in equation 9, becomes larger. However if there exists a large value of $\alpha_{2}, \delta_{m}$ may actually decrease as $\alpha_{1}$ is reduced.

Second-Order Momentum Compaction Factor. Assuming $\alpha=\alpha_{1}+\alpha_{2}\left(\Delta E / E_{0}\right)$ then at small values of $\alpha_{1}, \delta_{m}$, is defined as (10) 


$$
\delta_{m}=\left|\frac{a_{1}}{a_{2}}\right| \text {. }
$$

As $\alpha_{1}$ is decreased then $\delta_{m}$ will decrease. Fortunately $\alpha_{2}$, can be adjusted by changing sextupole magnet fields $(10)$ :

$$
\Delta \alpha_{2}=-\frac{\Delta S_{l}}{L_{0}}\left(\eta_{S x}^{3}-3 \eta_{S_{x}} \eta_{s y}^{2}\right)
$$

where $\Delta S_{l}$ is the change in integrated sextupole magnet, and $\eta_{S_{x}}$ and $\eta_{S_{y}}$ are the horizontal and vertical dispersion at the location of the sextupole. So by changing the sextupole magnetic fields, $\alpha_{2}$ can be made arbitrarily small permitting smaller values of $\alpha_{1}$.

Zeroeth-Order Momentum Compaction Factor. The zeroeth-order momentum compaction factor, $\alpha_{0}$, also effects the longitudinal dynamics. It shifts the energy at which particles making large amplitude excursions oscillate - moving these particles closer to the energy aperture. The zeroeth-order momentum compaction factor is given as (11)

$$
\alpha_{0}=-\frac{\left(\nu_{x}^{\prime} J_{x}+\nu_{y}^{\prime} J_{y}\right)}{L_{0}}
$$

where $\nu_{x}^{\prime}$ and $\nu_{y}^{\prime}$ are the horizontal and vertical ring chromaticities and $J_{x}$ and $J_{y}$ are the horizontal and vertical actions of the particle. Since sextupole magnets can change the ring's chromaticity, $\alpha_{0}$ can also be made zero by changing sextupole magnetic fields.

In addition, for positive $\alpha_{1}$, the head-tail instability (12) is suppressed if $\nu_{x}^{\prime}$ and $\nu_{y}^{\prime}$ are zero or slightly positive. Without sextupoles, $\nu_{x}^{\prime}$ and $\nu_{y}^{\prime}$ are always negative so the head-tail instability can limit the amount of current that can be stored in a single bunch.

Even though in principle it is possible to control $\alpha_{0}, \alpha_{2}, \nu_{x}^{\prime}$ and $\nu_{y}^{\prime}$ simultaneously with sextupoles, it is not always possible to do so in existing storage rings. In some rings there are either not enough sextupole families or the that the ones which exist are not located in regions of large dispersion.

\section{Effects of Magnet Field Errors and Power Supply Jitter}

We have already discussed how $\alpha_{1}$ and $\alpha_{2}$ are effected to first-order by changes in quadrupole and sextupole field strengths (equations 8 and 11). It is desirable to introduce one more expression which describes the effects of dipole errors on $\alpha_{0}$. Let's assume that there is an dipole field error in the ring which produces a horizontal angular kick, $\theta_{x}$, and a vertical angular kick, $\theta_{y}$. Then if the horizontal and vertical dispersion is $\eta_{\theta x}$ and $\eta_{\theta_{y}}$ at the location of the dipole respectively, there will be a change in the total pathlength for the on-energy particles of

$$
\frac{\Delta L}{L_{o}}=\Delta \alpha_{0}=\frac{1}{L_{o}}\left(\theta_{x} \eta_{\theta x}-\theta_{y} \eta_{\theta y}\right) \text {. }
$$

Using equations 8,11 , and 13 we can now examine the effects of magnetic field errors.

Dipole Errors. In the absence of an RF cavity, equation 13 states that dipole errors will increase the total path length of the closed orbit. But with an RF cavity present, the RF cavity frequency fixes the total path length so if there is a dipole 
error, the beam energy must change in order to keep the path length constant. The resulting change in energy is

$$
\frac{\Delta E}{E_{0}}=-\frac{1}{L_{0} \alpha_{1}}\left(\theta_{x} \eta_{\theta_{x}}-\theta_{y} \eta_{\theta_{y}}\right) \text {. }
$$

This means that at small values of $\alpha_{1}$, dipole kicks can give rise to large energy shifts which will in turn give rise to orbit shifts in regions of dispersion. At a given value of $\alpha_{1}$, the amount of a dipole error which can be tolerated will depend upon the physical aperture of the machine. The most harmful effect comes from dipole errors in regions of large dispersion.

Similarly to dipole errors, the effect of changes in the RF cavity frequency will also cause the energy to change (equation 15). By monitoring orbit changes in regions of large dispersion and feeding back on the RF frequency it is possible to stabilize the beam energy $(13,14)$.

Quadrupole Errors. From equation 8 one sees that quadrupole power supply fluctuations will change the value of $\alpha_{1}$. If $\alpha_{1}$ passes through zero, the beam will become unstable. So the amount of quadrupole field change and thus change in $\alpha_{1}$ that can be tolerated will depend upon how small $\alpha_{1}$ is. Field errors for quadrupoles located in regions of large dispersion will be the most harmful. By monitoring changes in the synchrotron tune and feeding back on a quadrupole power supply, it may be possible to stabilize $\alpha_{1}$ (14).

Sextupole Errors. From equation 8 one sees that sextupole power supply fluctuations will change the value of $\alpha_{2}$. The amount of variation in $\alpha_{2}$ that can be tolerated will depend upon the size of the energy aperture (equation 10).

It may be necessary to either impose strict limits for power supply jitter of dipole, quadrupole, and sextupole magnets located in regions of large dispersion or to use some feedback mechanisms such as those discussed above to stabilize the longitudinal motion. Let's now discuss how in practice it is possible to determine $\alpha_{1}$ and $\alpha_{2}$ in a storage ring.

\section{WHAT CAN WE MEASURE?}

\section{The first-order momentum compaction factor, $\alpha_{1}$}

There is no direct way of measuring the size of $\alpha_{1}$. There are however several indirects ways. It is possible to measure the synchrotron tune, the bunch length and the change in the beam orbit as a function of the change in RF frequency and then infer $\alpha_{1}$.

\section{Measurement of the Synchrotron Tune, $\nu_{s}$}

The most straight forward and easiest method to measure $\alpha_{1}$ is to measure the synchrotron tune (equation 4). The value of $\alpha_{1}$ can then be extracted assuming values for the RF frequency, peak voltage, synchronous phase, and electron energy. This measurement of $\nu_{s}$ should be done at low currents in order to minimize the effects of potential-well distortion.

\section{Measurement of the Bunch Length, $\sigma_{b}$}

By measuring the bunch length, $\sigma_{b}$, in addition to $\nu_{s}$ it is possible to deduce the value of $\alpha_{1}$ (equation 3) assuming some value for the relative energy spread of the beam. Because of the uncertainty of the energy spread this method is less straight forward than measuring the synchrotron tune. 


\section{Measurement of the Horizontal Beam Displacement, $\Delta x$}

A third way of measuring $\alpha_{1}$ is by measuring the horizontal beam displacement as a function of the relative change in if frequency, $\Delta f_{R F} / f_{R F}$. The displacement, $\Delta x$, is related to $\Delta f_{R F} / f_{R F}$ and can be written as

$$
\Delta x=\eta_{x} \frac{\Delta E}{E_{\mathrm{o}}}=\frac{\eta_{x}}{\alpha} \frac{\Delta L}{L}=-\frac{\eta_{x}}{\alpha} \frac{\Delta f_{R F}}{f_{R F}},
$$

where $\eta_{x}$ is the the horizontal dispersion. By looking at the beam displacement at some beam position monitor (BPM), it is possible to deduce $\alpha_{1}$. It is necessary to assume some value for $\eta_{x}$ at the BPM and since it is not possible to measure the dispersion directly, one must derive it using a model of the ring.

This last method has one added feature which the others do not have. It is possible to also determine the sign of $\alpha_{1}$. In the other techniques it is only possible to determine the magnitude of $\alpha_{1}$ but they can not specify whether $\alpha_{1}$ is positive or negative.

\section{Second-order momentum compaction factor, $\alpha_{2}$}

If $\alpha$ has higher-order momentum compaction terms, such as $\alpha_{2}$, then the value of the $\alpha_{1}$ measured will be energy dependent.

$$
\begin{aligned}
\left.\alpha_{1} \text { (measured }\right) & =\frac{d\left(\Delta L / L_{\circ}\right)}{d\left(\Delta E / E_{0}\right)} \\
& =\left.\alpha_{1}\right|_{\left(\Delta E / E_{0}=0\right)}+\left.2\left(\Delta E / E_{0}\right) \alpha_{2}\right|_{\left(\Delta E / E_{\circ}=0\right)}+\ldots
\end{aligned}
$$

If there exists a large $\alpha_{2}$ term then the synchrotron tune will be a function of the RF cavity frequency (17) and can be written as

$$
\nu_{s}=\nu_{s o}\left(1-4 \frac{\alpha_{2}}{\alpha_{1}{ }^{2}} \frac{\Delta f_{R F}}{f_{R F}}\right)^{1 / 4}
$$

where $\nu_{\text {so }}$ is the value of the synchrotron tune at $\Delta f_{R F}=0$. Therefore by measuring $\nu_{s}$ verses $\Delta f_{R F}$ it is possible to deduce the value of $\alpha_{2}$.

Incidentally, because the change in $\alpha_{2}$ as a function of $S_{l}$ is cubic function of dispersion (equation 11), it was suggested by Etienne Forest that in the case of small $\alpha_{1}$, measuring $\alpha_{2}$ verses sextupole strength would be an accurate way of measuring the value of the dispersion at the location of the sextupole. Lets now. briefiy discuss the history of low alpha (small $\alpha_{1}$ ) experiments in electron storage rings.

\section{HISTORY OF EXPERIMENTS}

The first low alpha experiments on electron storage rings began about 10 years ago at two second generation synchrotron light sources: the BESSY-1 ring in Berlin and also at the VUV ring at BNL (15). In both of these initial experiments little attention was given to controlling the values of $\alpha_{0}$ and $\alpha_{2}$. Nevertheless, in 1991 BESSY-1 (16) achieved a value of $\alpha_{1}$ of about 0.0013 (a factor of 12). However at this value of $\alpha_{1}$ the stored single bunch current was small and the lifetime was poor. In 1991 experiments began at the UVSOR ring at IMS $(17,18)$. In these experiments, in addition to reducing $\alpha_{1}, \alpha_{2}$ was also reduced using sextupoles. 
This allowed them to reduce $\alpha_{1}$ from 0.035 to 0.0002 (1a factor of 150). In 1993 low alpha experiments began at a furst generation light source, the SPEAR ring at SSRL (19). During these experiments beam was injected and stored in a negative $\alpha_{1}$ lattice (20). In 1994 experiments began at the Super-ACO ring at LURE $(21,22)$ that resulted in a reduction of $\alpha_{1}$ by a factor of 100 . Recently they demonstrated that at negative $\alpha_{1}$, with all sextupoles turned off, it was possible to store very large peak currents. In 1995 experiments have begun at two third generation light sources, ESRF (23) and ALS (24) both achieving short bunch lengths.

With the exception of SPEAR, all of the ring magnetic lattices are either of one of two types: double bend achromats (VUV, UVSOR, Super-ACO and ESRF) and triple bend achromats (BESSY-1 and ALS). Within each lattice type the techniques for achieving low alpha are similar. We will now discuss in some detail the results of two double bend achromats, UVSOR and Super-ACO. The results from ESRF are discussed by Laclare (23) and those from ALS are discussed by Robin et al (24) in other papers in these proceedings.

\section{DOUBLE BEND ACHROMATS}

The double bend achromat (DBA) lattice easily lends itself to low $\alpha_{1}$ operation. Of all of the existing storage ring lattices, the DBA requires the least amount of modification of the quadrupole fields and thus twiss parameters to achieve low $\alpha_{1}$.

\section{UVSOR}

The UVSOR lattice is a DBA which is made up of 4 DBA cells. A typical cell can be seen in figure 1. In UVSOR's normal- $\alpha_{1}$ configuration (figure 1 (a)), the dispersion function is slightly positive in the straight sections on the outside of the Bs (the bends) and is also positive in the straight sections between the Bs. In UVSORs zero- $\alpha_{1}$ configuration (figure 1 (b)). the quadrupole fields are adjusted to make the dispersion function on the outside of the Bs negative. The dispersion function passes from negative to positive value within the Bs making the integral in equation 6 equal to zero.

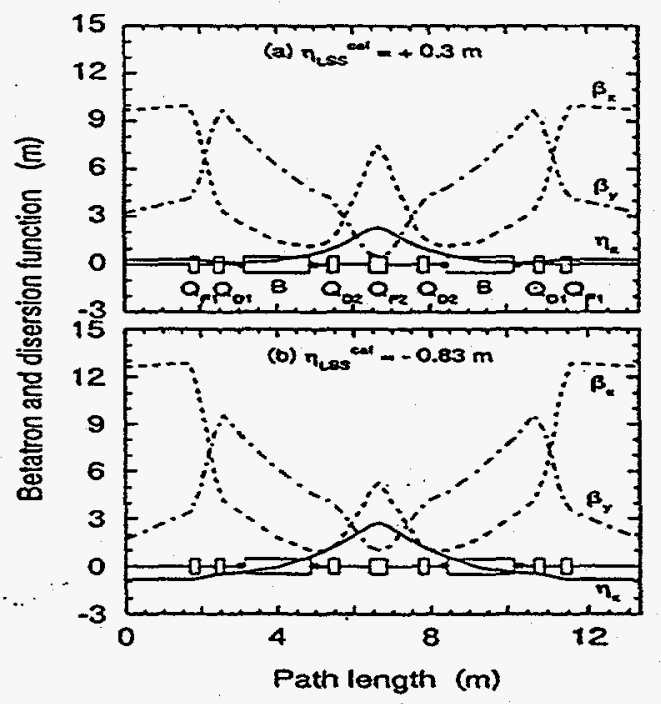

FIG. 1. Calculated Twiss parameters for one cell of the UVSOR lattice. a: Normal lattice configuration (top). b: Zero $\alpha_{1}$ configuration (bottom) 
In the UVSOR experiment, beam was injected into the normal- $\alpha_{1}$ lattice. Then as $\alpha_{1}$ was reduced, the quadrupoles were adjusted keeping the two tunes constant. Figure 2 shows the calculated values of $\alpha_{1}$ and the quadrupole strengths verses, $\eta_{L S S}$, the dispersion function in the center of the outside straight section. From the figure it is clear that there exist a smooth path from the normal- $\alpha_{1}$ to zero- $\alpha_{1}$ lattice.

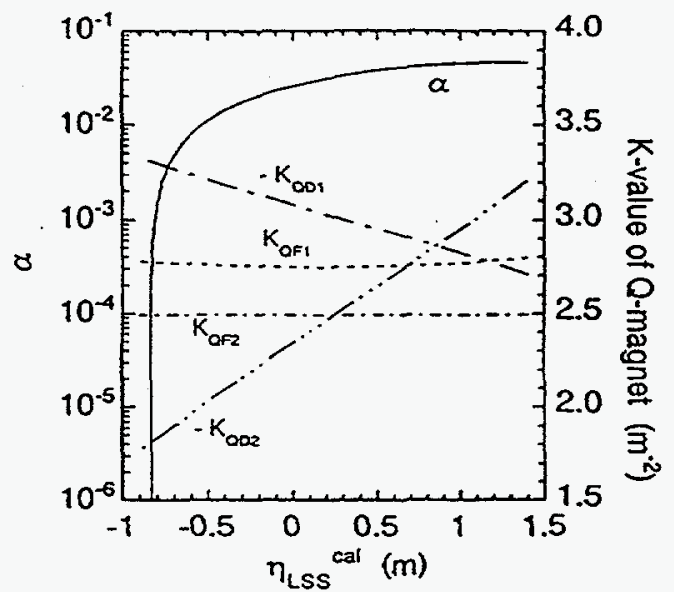

FIG. 2. Calculated $\alpha_{1}$ and $K$-values of the quadrupoles as a function of $\eta$ ws, the dispersion in the center of the outer straight section.

A very attractive feature the DBA is with the exception of $\alpha_{1}$, the normal $\alpha_{1}$ and zero- $\alpha_{1}$ lattice are very similar. Properties such as the Twiss parameters and the natural chromaticities are nearly identical. The emittance is only twice as large. The difference in the dynamics lies primarily in the longitudinal plane.

\section{Initial Measurements}

Initial experiments were made in 1991 and 1992. During these experiments the synchrotron tune was measured by exciting synchrotron oscillations with a phase shifter in the RF system. Throughout the experiments the RF voltage remained constant at $46 \mathrm{kV}$. Also the sextupoles were adjusted to keep the chromaticity zeroed in both planes.

The lowest value of $\alpha_{1}$ that was achieved was 0.0009 . At this value of $\alpha_{1}$ it was not possible to store more than $0.1 \mathrm{~mA}$ of current in a single bunch. What prevented $\alpha_{1}$ from being reduced further was that the energy acceptance of the ring, $\delta_{m}$; was becoming small due to a large value of $\alpha_{2}$ (equation 10). So in order to proceed further it was necessary to first measure $\alpha_{2}$ and then set it to zero.

\section{Measuring and Controling $\alpha_{2}$}

The size of $\alpha_{2}$ was determined by varying the RF frequency and measuring the synchrotron tune (figure 3). It was determined using equation 17 to be -0.15 . This meant that $\delta_{m}=\left|\alpha_{1} / \alpha_{2}\right|=0.6 \%$ which is rather small. A short Touschek lifetime (9) is what is most likely limiting the single bunch current.

The dependence of $\alpha_{2}$ on the strength of the SF family was also measured (figure 3 ). Using the calculated value of dispersion at the SF sextupoles, $\eta=2.3 m$, there is good agreement between the measurement and equation 11. Experimentally it 
was determined that the best setting for SF to minimize $\alpha_{2}$ was $86 \%$ of its initial value $\left(I_{S F}=0.45\right)$. Once $\alpha_{2}$ was reduced, it was possible to reduce $\alpha_{1}$ further. The smallest value of $\alpha_{1}$ which was obtained was 0.0002 . At $\alpha_{1}=0.0002, \sigma_{b}$ was measured to be 7.5 ps. At this small value of $\alpha_{1}$ it was not possible to store more than $0.1 \mathrm{~mA}$ of current in a single bunch.

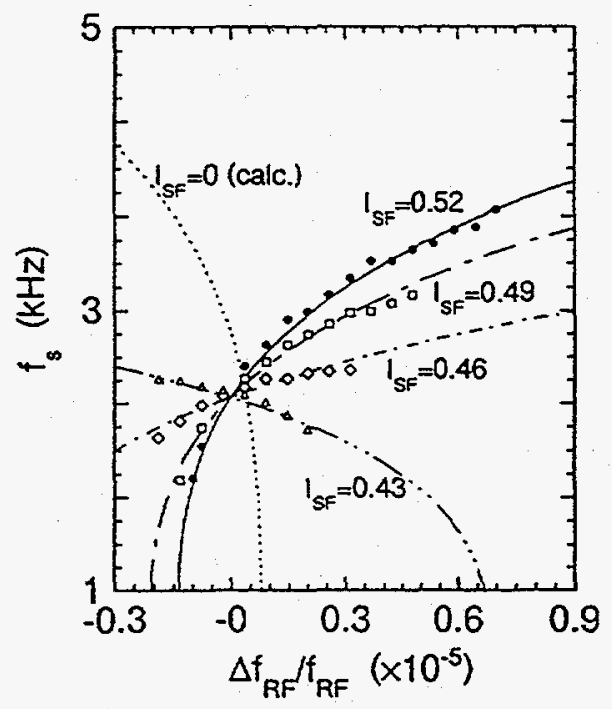

FIG. 3. Measured synchrotron oscillation frequency, $f_{s}$, as a function of the RF frequency for various strengths of the SF sextupoles. (In the initial measurements the value of SF was $I_{S F}=0.52$.)

Measurement of the bunch length verses synchrotron frequency can be seen in figure 4 . The bunch length is clearly proportional to the synchrotron frequency. The bunch profile can be seen in figure 5 for the normal and the lowest value of $\alpha_{1}$.

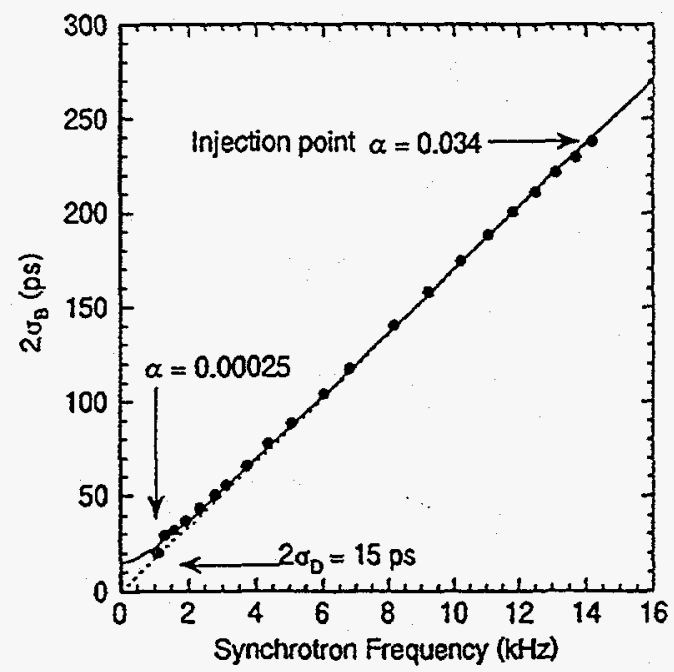

FIG. 4. Measured bunch length $\left(2 \sigma_{b}\right)$ plotted as a function of $f_{s}$ that was measured simultaneously. The solid line is a fit including the detector resolution. 


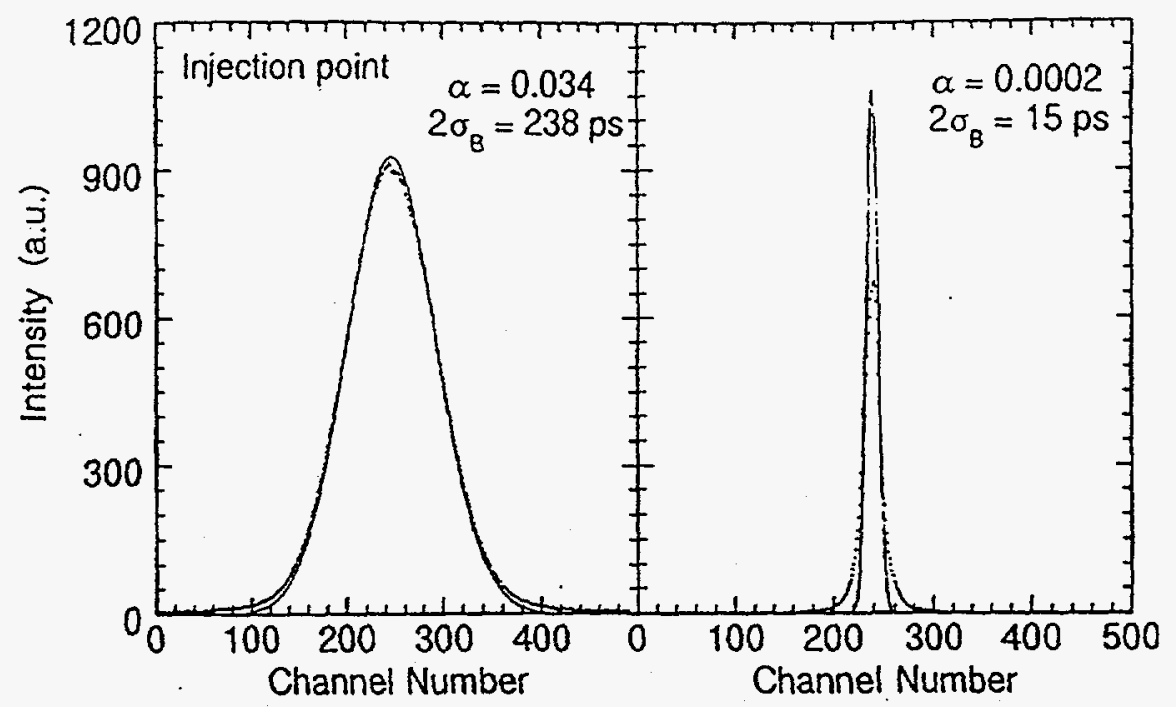

FIG. 5. Time profiles of the bunch measured by a steak camera at the normal operation condition (left) and at the lowest value of $\alpha_{1}$ (right).

\section{Current Dependent Bunch Lengthening}

The current dependent bunch length, $\sigma_{b I}$, was measured at currents ranging from several tens of $\mathrm{mA}$ to less than $0.1 \mathrm{~mA}$. The measurement was done for three different values of $\alpha_{1}$ and the result can be seen in figure 6 .

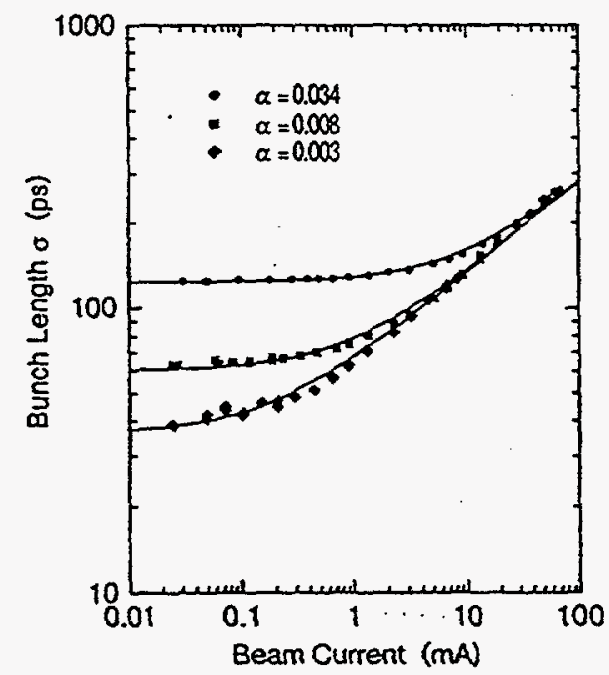

FIG. 6. Current dependence of the bunch length at three different values of $\alpha_{1}$. The lines indicated the results of the fit with equation 18 .

The microwave instability (25), which increases the energy spread of the bunch, was ruled out as the primary cause of the bunch lengthening for two reasons: In a 
previous experiment, no increase in the energy spread was observed when measuring the current dependence of the FEL gain (26) and the microwave instability has a. sharp threshold that is not seen in the data (figure 6). The cause of the bunch lengthening was determined to be potential-well distortion.

Based on the potential-well distortion theory (27), the bunch lengthening can be expressed as

$$
I=(2 \pi)^{-3 / 2} \frac{e T_{o}}{R^{3}} \frac{d V_{R F}}{d t} \frac{1}{[Z / n]_{e f f}}\left(\sigma_{b I^{3}}-\sigma_{b I} \sigma_{b o}^{2}\right),
$$

where $\sigma_{b I}$ is the bunch length at the current $I, \sigma_{b o}$ is the zero current bunch length, and $[Z / n]_{e f f}$ is the effective longitudinal coupling impedance. Two free parameters, $[Z / n]_{e f f}$ and $\sigma_{b o}$, were used to fit the data with equation 18 . The resulting values of $\sigma_{b 0}$ were in good agreement with the the deduced values from the synchrotron tune. The best fit (solid lines in figure 6) corresponds to a constant value of $[Z / n]_{e f f}$ of about $2.5 \Omega$. Due to the goodness of the fit it appears as if $[Z / n]_{e f f}$ is nearly constant over the range of bunch lengths measured. In general $[Z / n]_{\text {eff }}$ is not constant but a function of the length of the bunch.

\section{SUPER-ACO}

The standard lattice of the Super-ACO ring is also a DBA structure. For this particular experiment, large emittance detuned optics (figure 7) is used giving an initial $\alpha_{1}$ of 0.0015 . Each quadrupole has extra coils which can be powered to produce a sextupole field. This means that Super-ACO has 4 families of sextupoles.

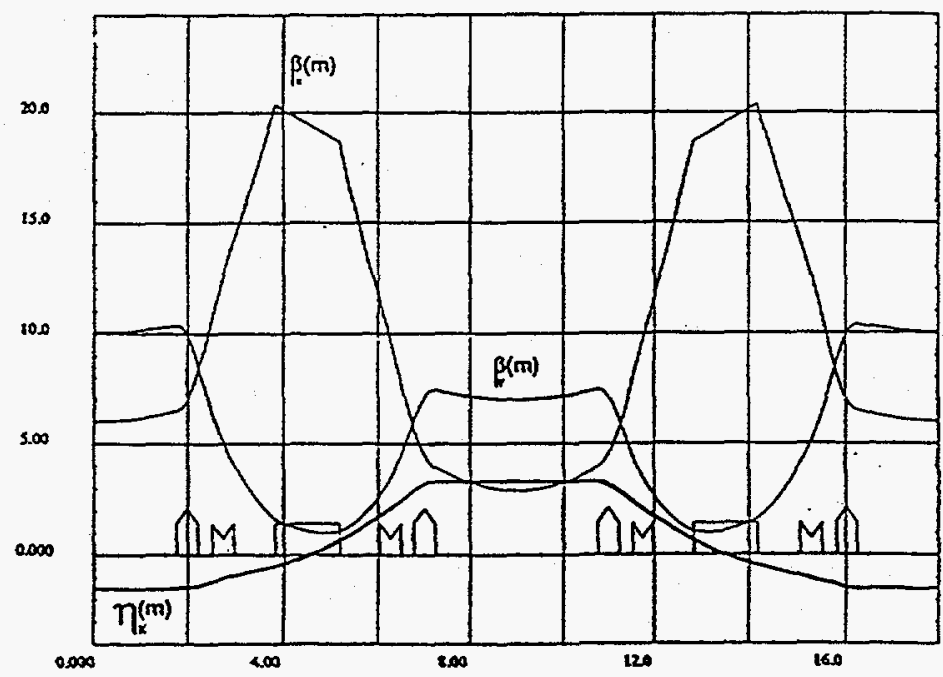

FIG. 7. Calculated Twiss parameters for one cell of the detuned Super-ACO lattice where $\alpha_{1}$ is adjusted to 0.00015 (100 times smaller than the initial value).

\section{Reducing $\alpha_{1}$}

The beam was injected at the large emittance operating point, then $\alpha_{1}$ was re duced step by step by changing the strength of the quadrupoles. During the descent, 
the synchrotron frequency was measured for each point of the path. Every time, the machine arrived at the operating point corresponding to the calculated value of $\alpha_{1}=0.003$, some difficulties appeared in terms of various instabilities, large closed orbit shift, poor beam lifetime and sudden beam losses. The variation of the synchrotron frequency which is linear with the square root of the calculated $\alpha_{1}$ down to $\alpha_{1}=0.003$ becomes nonlinear below this value (figure 8 ). Equations 16 and eq:measa2 show that for low values of $\alpha_{1}$, the term involving $\alpha_{2}$ can no longer be neglected. The three curves of figure 8 corresponds to different sets of sextupole strengths. One of these sextupole configurations (dark circles) reduced $\alpha_{2}$ to a nearzero value leading to a much lower value of the synchrotron frequency for the same calculated $\alpha_{1}$. At the lowest point, $\alpha_{1}=0.00015$ (100 times smaller than the initial value) the single bunch current was $0.1 \mathrm{~mA}$. This should correspond to a reduction of the natural bunch length by a factor of 10 . Unfortunately, the short electrode we use routinely for bunch length measurements does not permit to measure lengths less than 50 ps. Therefore no bunch length measurement was done for $\alpha_{1}$ smaller than 0.0036 .

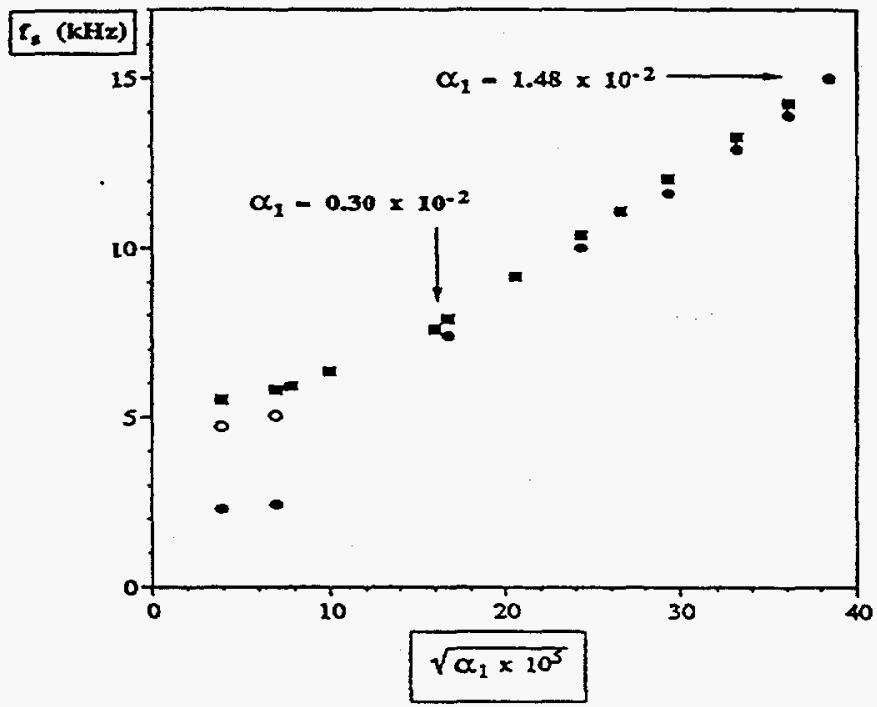

FIG. 8. Measured synchrotron frequency verses calculated $\alpha_{1}$ for three different values of sextupole strengths.

Systematic measurement and control of $\alpha_{2}$ were performed for one particular point of the descent path corresponding to $\alpha_{1}=0.00119$. As at UVSOR, the synchrotron frequency was measured as a function of the RF frequency for different values of the SX4 focusing sextupole family strength. Experimental values of $\alpha_{1}$ and $\alpha_{2}$ are then deduced by fitting equation 17 . Reducing the SX 4 strength by $5 \%$ from its nominal value brought the measured $\alpha_{2}$ to nearly zero $(-0.007)$. At this point, the synchrotron frequency was $4.2 \mathrm{kHz}$ which confirms the reduction of $\alpha_{1}$ by a factor 13 since at the injected point the synchrotron frequency is $15 \mathrm{kHz}$. The variation of $\alpha_{2}$ with the SX4 sextupole strength is linear as predicted by equation 11 . Using the experimental value of $\left(\Delta \alpha_{2}\right) /(\Delta S X 4)$ and equation 11 , the value of the dispersion in SX4 is deduced. It is about $3.1 \mathrm{~m}$ and agrees very well with the model calculated value of $3.2 \mathrm{~m}$. The compensation of $\alpha_{2}$ at this point of the path allowed us to store a single bunch of $5 \mathrm{~mA}$ with corrected chromaticities and beam lifetime longer 
than 10 hours. No bunch length measurements were performed in this conditions. Nonetheless the bunch lengthening curves as a function of current, measured for $\alpha_{1}$ $=0.0036$ and $\alpha_{1}=0.0148$, show that there is no effect of $\alpha_{1}$ on bunch length above $4 \mathrm{~mA}(21)$.

\section{Negative $\alpha_{1}$}

Super-ACO succeeded in storing beam in a lattice where $\alpha_{1}$ was negative. Because no head-tail instability exists when both $\alpha_{1}$ and the chromaticity are negative, the sextupoles were turned off leading to negative chromaticity in both plans. The stored beam current in a single bunch was above $100 \mathrm{~mA}$. In this configuration $\alpha_{1}$ was about -0.012 which is nearly the same absolute value as $\alpha_{1}$ for standard operation. Figure 9 shows the variation of the measured bunch length as a function of current. The lengthening with current is much slower than with positive $\alpha_{1}$. This result confurms the theoretical predictions of S.X. Fang et al. (5). Preliminary observations of bunch shapes also seem to agree with these simulations (figure 10).

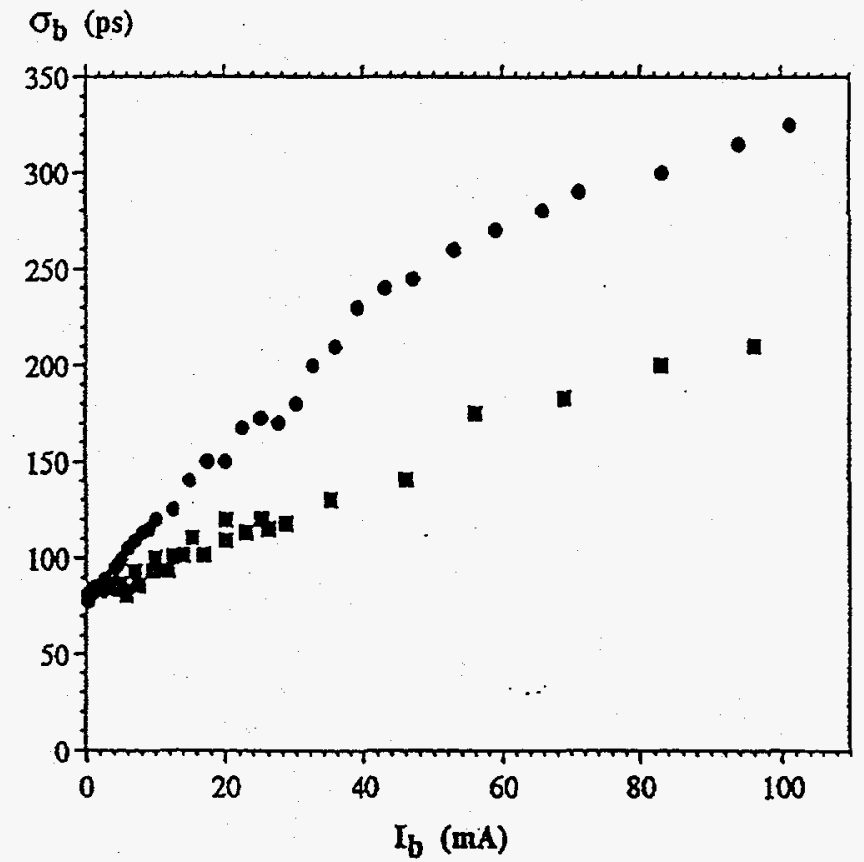

FIG. 9. Measured current dependent bunch lengthening at $\alpha_{1}=0.00148$ (circles) and $\alpha_{1}=-0.0012$ (squares). 


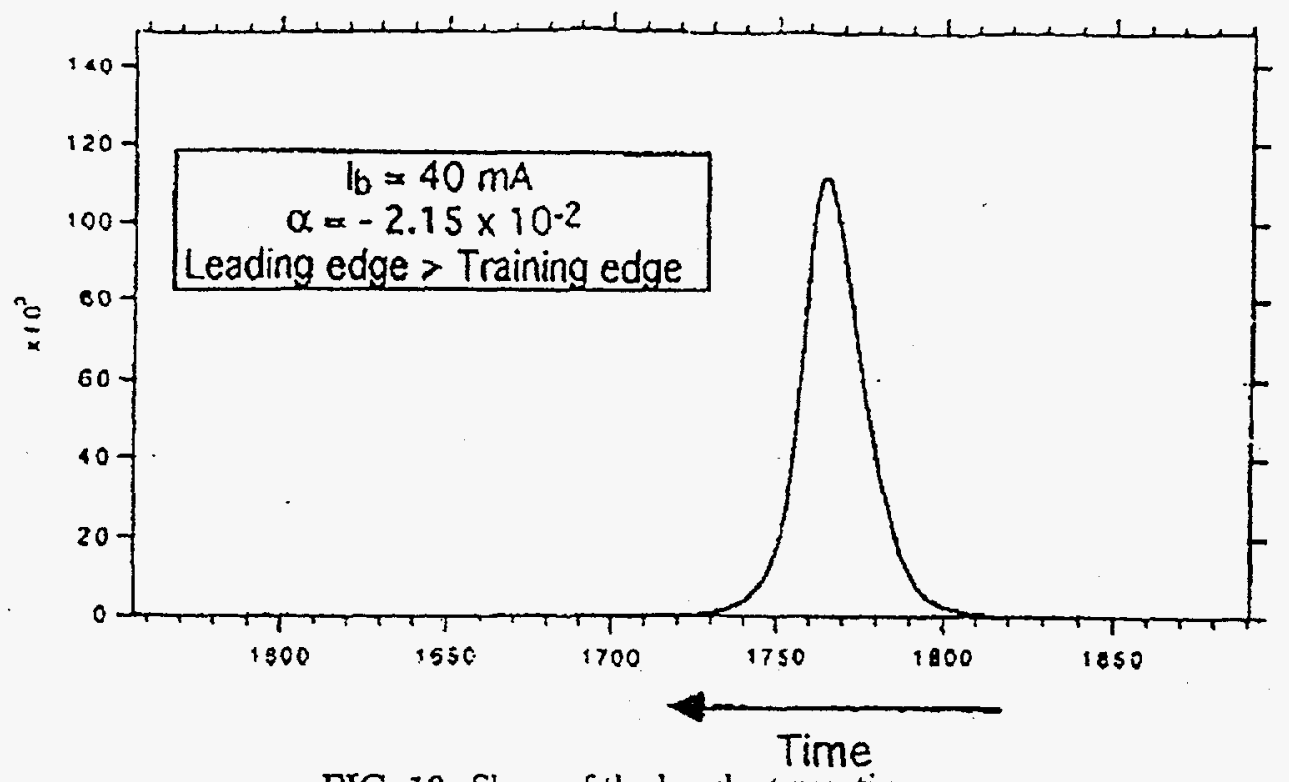

FIG. 10. Shape of the bunch at negative $\alpha_{1}$.

However as in the case of positive $\alpha_{1}$, experiments with different negative $\alpha_{1}$ values show that the bunch lengthening threshold is decreased when $\alpha_{1}$ is reduced and for operational intensities ( $>5 \mathrm{~mA}$ ) the bunch length is independent of the magnitude of $\alpha_{1}$ (figure 11). These bunch length measurements were made with a streak camera.

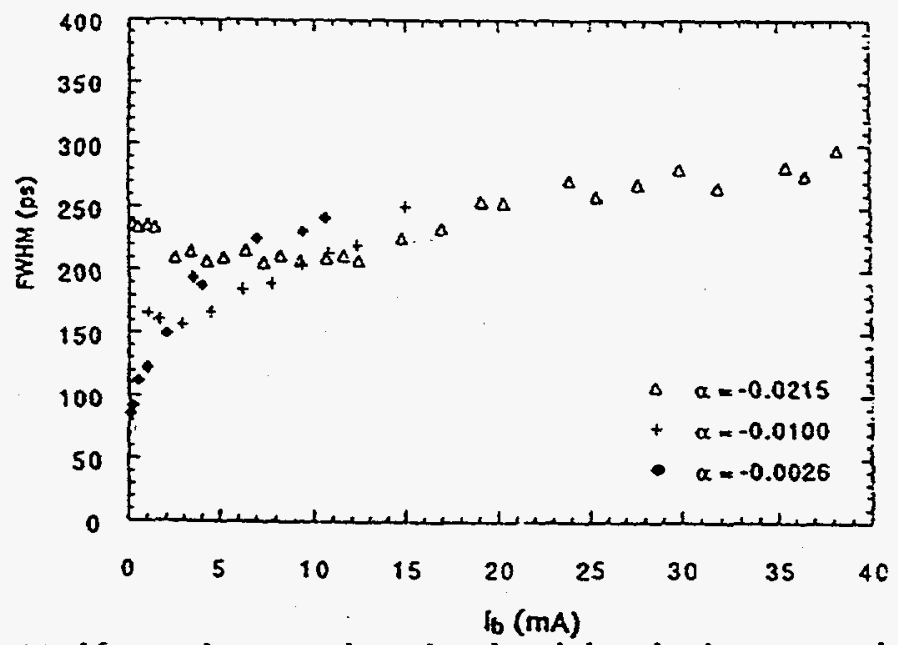

FIG. 11. Measured current dependent bunch leagthening at negative $\alpha_{1}$.

There are plans to do more detailed studies on bunch lengthening behavior with negative $\alpha_{1}$ on Super-ACO.

\section{SUMMARY OF RESULTS}

There have been some substantial advances in reducing the bunch lengths in synchrotron light sources by reducing the momentum compaction factor. By controlling higher-order momentum compaction factor terms, it has been possible to 
reduce the momentum compaction by 2 orders of magnitude resulting in bunch length reductions of a factor of 10 to less than 10 ps. Presently the amount of current that has been stored is at these lowest values of $\alpha_{1}$ is small, less than $1 \mathrm{~mA}$. A possible limit to the current may be the head-tail instability but it is not certain. Bunch lengthening has also been observed and has been attributed to potential-well distortion.

In addition recent experiments at Super-ACO show that negative $\alpha_{1}$ operation has achieved less bunch lengthening and higher peak currents than positive $\alpha_{1}$ operation, without cluromaticity compensation.

\section{Acknowledgements}

We wish to thank Alan Jackson, Albert Hofmann, Hideaki Ogaki, Etienne Forest, and Hiroshi Nishimura for many helpful comments.

\section{REFERENCES}

1. L. I. Schiff, Rev. of Sci. Instr. 17 (1946) 6.

2. J. S. Nodvick and D. S. Saxton, Phys.Rev. 96 (1954) 180.

3. A. Hofmann, CERN LEP-TH Note 4 (1982).

4. C. Pellegrini and D. Robin, Nucl. Instr. and Meth. in Phys. Res. A301 (1991) 27-36.

5. S.X. Fang et al, KEK Preprint 94-190 (1995).

6. D.A.G. Deacon, Phys. Rep. 76 (1981) 349.

7. We should mention that there have been a number of related studies on transition crossing in hadron synchrotrons which are not discussed in this paper.

8. M. Sands, "The Physics of Electron Storage rings: an Introduction", Stanford Linear Accelerator Note, SLAC-121, (1970).

9. J. LeDuff, Nucl. Instr. and Meth. A.239 (1985) 83.

10. D. Robin et al, Phys. Rev. E 48,3 (1993) 2153.

11. Formula derived by Etienne Forest, private communication.

12. See for instance R. Ruth, Accelerator Physics Issues for Super Conducting Super Collider, Ann Arbor, 1983, ed. M. Tigner, Univerity of Michigan rep. no. UMHE 84-1 (1983) 151.

13. E. Ciapala et al, IEEE Transactions on Nuclear Science, NS-26, No. 3 (1979) 3571-3573.

14. Suggestion made by Albert Hofmann.

15. S. Krinsky et al., "Storage Ring Development at the NSLS", BNL-46615, (1991).

16. P. Kuske, private communication.

17. H. Hama, S. Takano and B. Isoyoma, Nucl. Instr. and Meth. in Phys. Res. A329 (1993) 29-36.

18. H. Hama et al. "Operation with the Low Momentum Compaction Factor on an Electron Storage Ring", Procedings of the 9th Symposium on Accelerator Science and Technology, Tsukuba, Japan (1993) 468.

19. P. Tran et al. "Status of the Variable Momentum Compaction Storage Ring Experiment in SPEAR ${ }^{n}$, Proceedings of the 1993 Particle Accelerator Conference, (1993) 173.

20. A. Hoffman, private communication.

21. A. Nadji et al, "Quasi-Isochronous Optics for Super-ACO", Proceedings of the 4th European Particle Accelerator Conference, (1994) 128.

22. A. Nadji et al, "Experiments with the Super-ACO storage ring operating in a quasi-isochronous mode ${ }^{n}$, to be published.

23. J. Laclare, "ESRF Experience of Short Bunches in Storage Rings", in this workshop's proceedings this workshop (1995).

24. D. Robin et al, "Low Alpha Experiments on ALS", presented at this workshop (1995).

25. D. Boussard, CERN-SPS Report Lab.II/RF/75-2 (1975).

26. S. Talano, H. Hama and G. Isoyama, Jpn. J. Appl. Phys. 32 (1993) 1285.

27. B. Zotter, Proc. CERN Accelerator School, Paris 1994, CERN 85-19 (198.5) 415. 